\title{
summary
}

\section{Chlorhexidine varnish may have a role in the management of dental caries in adults with dry mouth}

\author{
Bantin DW, Papas A, Clark DC, Proskin HM, Schultz, Perry R. The effectiveness of $10 \%$ chlorhexidine varnish treatment \\ on dental caries incidence in adults with dry mouth. Gerodontol 2000; 17:67-76
}

Question: Is chlorhexidine varnish effective in reducing caries in adults who have reduced salivary flow?

Objective To determine whether chlorhexidine varnish would reduce caries incidence in adults who have reduced salivary flow due to medication.

Design A multicentre randomised controlled trial conducted at three dental schools.

Intervention A two-stage application of either active (10\% chlorhexidine), sham (to mimic taste) or placebo varnish treatment was carried out once a week for 4 weeks by a dental hygienist, with a single re-application at 6 months. Clear inclusion and exclusion criteria were defined. If patients developed two or more carious lesions in any 2-month period or more than four carious lesions during the study period they were withdrawn.

Outcome measures Caries increment.

Results Of 540 screened patients, 240 were enrolled in study with 236 completing at least on the follow-up visit. Comparison of the active and placebo groups showed a significant reduction (see Table) in root caries increment of $41 \%$ and $25 \%$ in total caries increment. The $14 \%$ reduction in coronal caries did not reach clinical significance. A number of transient minor adverse events were noted.
Table 1 Mean (SD) caries increment

\begin{tabular}{lccc}
\hline & Active & Placebo & Sham \\
\hline Coronal caries & $1.79(2.36)$ & $2.09(2.13)$ & $1.83(2.34)$ \\
Root caries & $0.77(1.33)$ & $1.30(1.70)$ & $1.14(1.62)$ \\
Total caries & $2.56(3.05)$ & $3.39(3.52)$ & $2.97(3.22)$ \\
\hline
\end{tabular}

SD, Standard deviation

Conclusions The results suggest that $10 \%$ chlorhexidine varnish may have an important role to play in the management of dental caries in adults with dry mouth.

Evidence-Based Dentistry (2002) 3, 53. doi:10.1038/sj. ebd.6400116

Address for reprints: Dr DW Banting, Faculty of Medicine and Dentistry, School of Dentistry, University of Western Ontario, London, Ontario,

Canada N6A 5C1. E-mail: dbanting@julian.uwo.ca.

\section{Commentary}

This clinical trial indicates that a caries preventive programme consisting of five professional treatments with a $10 \%$ chlorhexidine varnish during a period of 12 months significantly reduces root caries increments and total caries increments in subjects with a dry mouth. The study applies the classic randomised, double-blind, placebo-controlled trial, although the report of the results is less stringent. For example, the authors do not give a detailed account of the subjects who were lost to follow-up. Furthermore, a description of the baseline caries experience in the intervention groups is lacking. This constitutes a potentially serious problem because the loss of subjects could be related to the baseline caries levels, thereby introducing a bias in the estimation of the effects.

The study gives the estimated effects on the development of caries but fails to measure the precision. A calculation of the $95 \%$ confidence limits demonstrates that caries reductions may be expected to vary between 0.05 and 1.01 surfaces/year for root caries and between -0.33 and 1.83 surfaces/year for total caries. It is questionable whether such small and variable caries reductions are clinically meaningful. In this context it should also be noted that $21 \%$ of the subjects in the chlorhexidine varnish group were withdrawn from the study because of high caries increments. For the uncritical reader the results of the present study may be taken to indicate that professional chlorhexidine varnish applications is the method of choice for controlling caries development in subjects who have a dry mouth. Because of the lack of a positive control group it is, however, not possible to say whether the present treatment is superior to other professionally administered treatments for this category of patient. In some studies, professional application of topical fluorides has proved to be at least as effective as chlorhexidine in controlling dental caries.

Bente Nyvad Royal Dental College, University of Aarhus, Aarhus, Denmark 